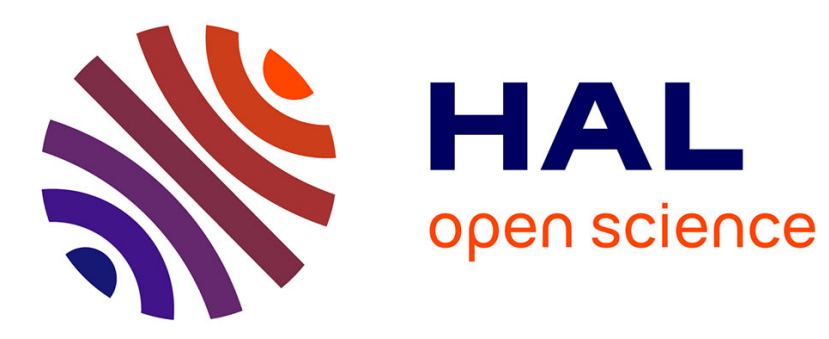

\title{
Sociétés médiévales et approches géographiques : un dialogue de sourds?
}

\author{
Nacima Baron-Yelles, Stéphane Boissellier
}

\section{To cite this version:}

Nacima Baron-Yelles, Stéphane Boissellier. Sociétés médiévales et approches géographiques: un dialogue de sourds?. XXXVIIIe congrès de la SHMESP, May 2007, Cergy-Pontoise, Marne-la-Vallée, Saint-Quentin-en-Yvelines, France. pp.269-297. halshs-00344288

\section{HAL Id: halshs-00344288 \\ https://shs.hal.science/halshs-00344288}

Submitted on 9 Feb 2017

HAL is a multi-disciplinary open access archive for the deposit and dissemination of scientific research documents, whether they are published or not. The documents may come from teaching and research institutions in France or abroad, or from public or private research centers.
L'archive ouverte pluridisciplinaire HAL, est destinée au dépôt et à la diffusion de documents scientifiques de niveau recherche, publiés ou non, émanant des établissements d'enseignement et de recherche français ou étrangers, des laboratoires publics ou privés. 


\section{Sociétés médiévales et approches géographiques : un dialogue de} sourds?

Madame Nacima Baron-Yellès, Monsieur Stéphane Boissellier

Citer ce document / Cite this document :

Baron-Yellès Nacima, Boissellier Stéphane. Sociétés médiévales et approches géographiques : un dialogue de sourds ?. In: Actes des congrès de la Société des historiens médiévistes de l'enseignement supérieur public, $38^{\mathrm{e}}$ congrès, Île de France, 2007. Etre historien du Moyen Age au XXle siècle. pp. 163-177;

doi : 10.3406/shmes.2007.1951

http://www.persee.fr/doc/shmes_1261-9078_2008_act_38_1_1951

Document généré le 04/06/2016 


\section{Sociétés médiévales \\ et approches géographiques: \\ un dialogue de sourds?}

Nacima Baron, Stéphane Boissellier

La réflexion épistémologique sur les rapports entre les sources textuelles et matérielles bat son plein depuis l'essor de l'archéologie médiévale ${ }^{1}$, et celle sur les rapports avec la sociologie et l'anthropologie n'a jamais vraiment cessé, avec des hauts et des bas, depuis la fondation des Annales. En regard, les liens entre histoire et géographie, souffrant du discrédit d'être une alliance d'origine académique, ne semblent plus guère susciter d'intérêt, tant le divorce paraît consommé : c'est vrai chez les médiévistes ${ }^{2}$, c'est également vrai chez certains géographes, selon lesquels l'association entre historiens et géographes ressemble à un "mariage usé " ". Est-il donc nécessaire de ressusciter les vieilles lunes de la "géographie historique" de Longnon ou même, plus récemment, de la "géohistoire " d'Higounet ${ }^{4}$ pour montrer la fécondité des croisements qui restent à développer? Notre réflexion ne sera pas facile, car la confrontation entre les approches des deux disciplines date surtout d'une époque où la réflexion épistémologique était moins avancée.

1. Notre réflexion peut d'ailleurs s'inspirer de G. Noyé et Fr. Bougard, "Archéologie médiévale et structures sociales. Encore un effort ", Liber largitorius. Études d'histoire médiévale offertes à Pierre Toubert par ses élèves, dir. D. Barthélemy et J.-M. Martin, Paris, 2003 (Hautes études médiévales et modernes, 84), p. 331-346.

2. Pour une réflexion épistémologique sur les rapports entre histoire et géographie, voir le recueil d'essais de H. C. Darby, The Relations of History and Geography. Studies in England, France and the United States, Exeter, 2002, ou, plus synthétique, A. R. H. BAKER, Geography and History. Bridging the Divide, Cambridge, 2003; l'absence d'ouvrages récents en français sur ce thème est significative, si l'on excepte: Où en est la géographie historique? Entre économie et culture, dir. P. Boulanger et J.-R. Trochet, Paris, 2005, qui est plus thématique qu'épistémologique et n’inclut que des géographes (!).

3. G. RoQues, Décrypter le monde aujourd'hui. La crise de la géographie, Paris, 2006, p. 121.

4. Voir Ch. Higounet, "La géohistoire", L'histoire et ses méthodes. Recherche, conservation et critique des témoignages, dir. Ch. Samaran, Paris, 1961, p. 68-91. 
Étant dans un colloque d'historiens, la question posée sera plutôt : qu'est-ce que le médiéviste peut attendre de la géographie? Il est bien entendu que cette question s'inscrit plus largement dans le contexte des sciences humaines et sociales, notamment par le fait que les méthodes de l'analyse spatiale des sociétés utilisées en "géographie humaine " sont très étroitement liées aux concepts sociologiques : en utilisant celles-là, les historiens adoptent du même coup ceux-ci. Il faut également préciser que nous ne parlerons ici que du point de vue des médiévistes qui utilisent principalement des sources écrites, en écartant l'usage des sources matérielles. Nous considérons, quant à nous, que la distinction entre ces sources n'a pas à être durcie, mais c'est la place faite dans ce colloque à l'approche spécifiquement archéologique qui justifie notre restriction. Celle-ci oriente d'ailleurs fortement notre approche, car les liens entre archéologues médiévistes et géographes sont beaucoup plus importants ou au moins plus évidents 5 . Plaider pour une inter-disciplinarité entre histoire médiévale et géographie sociale est une cause désespérée ou, tout au moins, un long chemin semé d'embûches. Nous présenterons quelques réflexions générales puis les problèmes pratiques.

\section{Rappel historique et problèmes de principes : pourquoi le médiéviste aurait-il besoin de concepts géographiques? \\ Les concepts spatiaux sont universels et transdisciplinaires}

Sans répéter le rapport introductif de Monique Bourin et Élisabeth Zadora$\mathrm{Rio}^{7}$ à notre précédent congrès sur l'organisation spatiale, il convient de

5. Un exemple d'utilisation croisée, par des géographes et des médiévistes, du concept de paysage - en particulier du paysage fluvial - a fait l'objet d'un séminaire interdisciplinaire co-organisé par Nacima Baron à Saumur, les 14 et 15 octobre 2004, dans le cadre de la "Zone Atelier Loire ", programme de recherche interdisciplinaire soutenu par le CNRS et publié à l'adresse suivante : http://www.msh.univ-nantes.fr/documentation/zal/Documents/ResumesSaumurV1. pdf. Voir aussi G. ChovQuer, Quels scénarios pour l'histoire du paysage? Orientations de recherche pour l'archéogéographie, Coimbra-Porto, 2007.

6. Une partie des réflexions qui suivent sont reprises de St. BoIssellier, "Introduction à un programme de recherches sur la territorialité : essai de réflexion globale et éléments d'analyse », De l'espace aux territoires: pour une étude de la territorialité des processus sociaux et culturels en Méditerranée occidentale médiévale, état des recherches, sources, objets et méthodes. Actes de la table ronde, Centre d'études supérieures de civilisation médiévale (Poitiers), 8-9 juin 2006, éd. St. Borssellier, Turnhout, 2008, à paraître.

7. M. Bourin et É. Zadora-Rio, "Pratiques de l'espace : les apports comparés des données textuelles et archéologiques ", Construction de l'espace au Moyen Age : pratiques et représentations, Paris, 2007 , p. 39-55. 
rappeler une évidence, qui peine encore à s'imposer. L'organisation dans l'espace est une modalité fondamentale des sociétés humaines (et des comportements de certaines espèces animales) : une société, qu'est-ce d'autre que le rapport, plus ou moins direct, entre un groupe humain (un agrégat social) et un lieu spécifique (une unité spatiale) ? Plus précisément, toute activité humaine s'inscrit dans une certaine étendue d'espace et la détermine autant qu'elle en est affectée ${ }^{8}$. L'espace est la modalité physique (le lieu) de rencontre et de combinaison de toutes les structures matérielles et symboliques que les diverses sciences humaines s'ingénient à isoler, il est l'« ingrédient " par lequel s'opère la complexité du réel. Comme le dit Fernand Braudel, l'" espace, source d'explication, met en cause à la fois toutes les réalités de l'histoire, toutes les parties prenantes de l'étendue : les États, les sociétés, les cultures, les économies" ». Pour reprendre un auteur géographe contemporain, l'" espace est la matière des relations sociales ${ }^{10}$ ". Naturellement, le facteur spatial est plus directement sensible à qui étudie le lien social et politique, et les activités humaines ayant une forte emprise matérielle : la démographie et le peuplement, les pratiques agricoles et les flux marchands, les paysages et la topographie des habitats, la formation des territoires politiques et administratifs. C'est dans ces domaines que le recours aux concepts et aux instruments élaborés par les géographes est le plus nécessaire.

Du point de vue de la configuration globale des sciences humaines, la prise en compte du facteur spatial doit nous rapprocher des approches géographiques récentes, sans forcément passer par l'intermédiation de l'anthropologie, qui est le mode d'approche le plus pertinent dans de nombreux domaines, comme l'imaginaire ou les économies anciennes, mais pas dans celui-ci ${ }^{11}$. En effet, la géographie est tout de même la science par excellence de l'espace social, ou au moins l'est-elle devenue avec la " nouvelle géographie " qui s'est constituée entre les années 1950 et 1970. De fait, à une époque d'impérialisme de la géographie physique, quand la

8. Le fondateur de la sociologie en France, Émile Durkheim, définit largement la « morphologie sociale " comme les formes spatiales d'un substrat humain. Certes, cette définition très géographique visait prosaïquement une alliance contre la toute-puissance académique de l'histoire, mais l'influence du grand géographe allemand Friedrich Ratzel sur Durkheim est par ailleurs incontestable.

9. F. Brauder, Civilisation matérielle, économie et capitalisme, $X V$-XVIIf siècle, t. 3, Le temps du monde, Paris, 1979, p. 12.

10. M. Lussault, L'Homme spatial. La construction sociale de l'espace humain, Paris, 2007.

11. On trouvera une historiographie de la notion d'espace social dans les différentes sciences humaines (notamment son étonnante et durable ignorance par la sociologie et l'anthropologie) dans G. Di MÉo, Géographie sociale et territoires, Paris, 1998, p. 32-35, ouvrage qui sera notre principale référence conceptuelle. 
géographie " humaine " était un simple « inventaire du monde » descriptif, un usage servile des approches géographiques en histoire - les " conditions naturelles " de tel phénomène - a conduit à un déplorable déterminisme et à une dichotomie entre espace et société. Ce défaut, spécialement et durablement présent dans les grandes monographies régionales médiévistes et modernistes, a détourné, voire dégoûté, les historiens de l'approche spatiale, les privant du même coup des apports récents de la géographie sociale. Pourtant, même le contact entre une histoire événementielle et une géographie déterministe a eu l'effet positif d'introduire les nécessaires préoccupations géostratégiques dans l'analyse du politique ${ }^{12}$.

À partir des années 1970, au moment où la géographie humaine achevait sa mue et avait enfin autre chose à offrir que des cadres, l'anthropologie historique a entrainé de nombreux chercheurs à aborder des domaines - la parenté, l'imaginaire, le corps, les rites, la monumentalité de l'écrit - dans lesquels la dimension spatiale était souvent présente mais rarement en tant que telle ${ }^{13}$, et traitée de toute façon fort abstraitement, catégorie analytique plutôt que descripteur du réel ${ }^{14}$. En effet, utilisant abondamment des sources "littéraires" (savantes ou savamment construites : contes, sermons, traités) pour y traquer les mécanismes des représentations (mythes, oralité, motifs folkloriques ou mémoire) en partant du principe que toute structure linguistique n'est qu'une médiation (même dans les documents les plus triviaux), les médiévistes anthropologues - dont les apports sont par ailleurs indéniables et considérables dans de nombreux domaines - ont parfois été conduits à adopter les concepts trop spéculatifs

12. Voir, par exemple, J. Brunhes et C. VAllaux, La géographie de l'histoire. Géographie de la paix et de la guerre sur terre et sur mer, $2^{\circ}$ éd., Paris, 1921.

13. En 1974, les 3 volumes qui constituent le « tournant critique " de la nouvelle histoire ne considèrent l'analyse spatiale ni comme un nouveau problème, ni comme une nouvelle approche, ni comme un nouvel objet, cf. Faire de l'histoire, dir. J. Le Goff et P. Nora, Paris, 1974; en 1978, La nouvelle histoire, dir. J. LE Goff, rééd. Bruxelles, 1988, n’aborde pas le problème de l'espace. Enfin, H.-J. SснміDт, "Espace et conscience de l'espace dans l'historiographie médiévale allemande", Les tendances actuelles de l'bistoire du Moyen Age en France et en Allemagne. Actes des colloques de Sèvres (1997) et Göttingen (1998) organisés par le CNRS et le Max-Planck-Institut für Geschichte, dir. J.-Cl. Schmitt et O. G. Oexle, Paris, 2002 (Histoire ancienne et médiévale, 66), p. 514-515, fair la même constatation pour la recherche médiévale allemande.

14. Dans leur synthèse historiographique, J. Berlioz et J. LE Goff, "Anthropologie et histoire ", L'histoire médiévale en France. Bilan et perspectives, dir. M. Balard, Paris, 1991, p. 269-303, abordent l'espace au Moyen Âge dans le paragraphe sur "Les systèmes de représentation " (oppositions sauvage/domestiqué et monde réelíau-delà). Quant à J. Le Goff et J.-C. SchmirT, "L'histoire médiévale ", Cahiers de civilisation médiévale, 39 (1996), p. 9-25, ils parlent d'une " spatialisation de la pensée " au Moyen Âge central, ce qui désigne probablement une plus grande intégration de la dimension spatiale dans la réflexion intellectuelle médiévale. 
des «littéraires " et des linguistes, imprégnés de structuralisme, voire des psychanalystes. On va voir les effets que l'intermédiation anthropologique a eus sur les conceptions spatiales des médiévistes.

\section{De l'usage des concepts spatiaux chez les médiévistes}

Quel est le degré de pénétration de la pensée géographique chez les médiévistes? Même dans les domaines traditionnels comme l'étude du peuplement, il faut bien avouer que les médiévistes - les historiens des textes plus que les archéologues ${ }^{15}$ - ont été réticents à la modélisation et à l'emploi de concepts géographiques " durs ", malgré la forte tradition d'emprunts aux géographes. C'est seulement en 1997 que la théorie de la centralité devient l'objet même d'une recherche médiéviste (sur un réseau urbain) ${ }^{16}$, alors qu'elle est un passage obligé de la réflexion géographique depuis des décennies. Il y a toutefois une brillante exception : depuis plusieurs décennies, les historiens de la morphologie urbaine ancienne n'hésitent pas à transposer les concepts géométriques des urbanistes et architectes. Travaillant sur des espaces restreints et denses, voire pleins, donc isotropes (en fait sur des "lieux»), où les structures sociales sont fortement matérialisées par des constructions, il leur est plus facile de concevoir l'espace comme une étendue physique continue et il semble donc plus légitime d'y appliquer des règles telles que la polarité, le plan directeur, l'autorégulation des formes (des parcelles ou des îlots) ${ }^{17}$. À l'échelle urbaine ou infra-urbaine,

15. Pour ces derniers, voir par exemple le récent L. Nunninger, L. Sanders, F. Favory, P. Garmy, C. Raynaud, C. Rozenblat, L. Kaddouri, H. Mathian et L. Schneider, " La modélisation des réseaux d'habitat en archéologie : trois expériences ", M@ppemonde, n 83 (2006/3), cf. http://mappemonde.mgm.fr/num 1 1/articles/art06302.html. Les pionniers du travail inter-disciplinaire avec les géographes et de la systématisation par des théories spatiales ont été, logiquement, les archéologues médiévistes travaillant sur les paléo-environnements, mais ils sont actuellement rejoints par leurs collègues travaillant plus classiquement sur les habitats.

16. J.-L. FraY, "Villa in media ". Réseau urbain et perception de l'espace. Essai d'application de la théorie de la centralité au réseau urbain de la Haute-Lorraine médiévale (début Xf-début XIV siècle), HDR histoire, univ. Paris 1, 1997. On peut voir aussi le mémoire également inédit de M. BochacA, Villes et structuration de l'espace en Bordelais, fin de l'Antiquité-milieu du XVI siècle, HDR histoire, univ. Lyon 2, 2000. Plus récemment, j'ai tenté (dans St. Borssellier, Le peuplement médiéval dans le Sud du Portugal. Constitution et fonctionnement d'un réseau d'habitats et de territoires. XIf-XV siècles, Paris, 2003, qui est aussi un mémoire d'HDR) une application systématique de certains concepts spatiaux (définis moins théoriquement - et donc moins rigoureusement - pour les adapter plus facilement au contexte) aux principales formes d'organisation sociale des territoires locaux (détroit juridictionnel, paroisse, terroir) et à la rériculation des lieux et territoires à l'échelle supra-locale.

17. Les villes italiennes, grâce à une documentation écrite exceptionnelle, fournissent les plus beaux exemples de cette approche. Sur des sources moins pléthoriques mais avec des approches pas moins rigoureuses, voir D. MENJoT, Murcie castillane. Une ville au temps de la frontière (1243-milieu du 
cette approche reste encore peu suivie dans les autres secteurs de la recherche médiéviste. En revanche, pour l'échelle supra-locale, la réflexion des historiens du fait urbain a souvent proposé des modèles (pas forcément suivis) à tous ceux qui s'interrogent sur la construction sociale et matérielle des grands territoires, et c'est de ce mouvement que sort la généralisation, d'ailleurs plus ou moins heureuse, de concepts tels que le rapport centrepériphérie, les aires (d'influence), les réseaux ${ }^{18}$ ou l'emboîtement. Cependant, il semble que ce soit encore à l'échelle locale, mais cette fois " hors les murs ", qu'ont été déployées, par les médiévistes urbanistes, les analyses spatiales complexes les plus rigoureuses, à propos de la formation matérielle des espaces socialement et culturellement dominés par les villes ${ }^{19}$, qui pourraient renouveler la notion de "région ".

Au-delà de problèmes techniques et conceptuels précis, il est indéniable qu'il y a eu, par un effet de mode, une certaine contamination de l'expression des médiévistes par le lexique géographique. Cela ne va pas sans poser des problèmes : outre les utilisations impropres de la spatialité20, certains de ceux qui étudient les implications spatiales des phénomènes sociaux acceptent l'idée avancée par certains géographes, selon laquelle l'espace est une "chose-en-soi ", en interprétant dans un sens réaliste la conception kantienne de l'espace comme une "forme a priori de la sensibilité $^{11}$ ". On peut prendre l'exemple du rapport fonctionnel " centre/ périphérie "; issu de la théorie allemande de la centralité - celle de Walter Christaller, après celle, toute symbolique, de Raymond Lulle! - et généralisé

XV siècle), Madrid, 2002, qui résume toutes les recherches sur les villes médiévales hispaniques. Dans ces ouvrages, d'amples références sont faites aux grands classiques de la théorie urbanistique appliquée aux temps anciens, tels que Lewis Mumford ou Pierre Lavedan. On trouvera une bonne historiographie des approches du fait urbain dans P. Boucheron et D. Menjot, "Livre 2. La ville médiévale ", Histoire de l'Europe urbaine, t. 1, De l'Antiquité au XVIIr siècle. Genèse des villes européennes, dir. J.-L. Pinol, Paris, 2003, p. 285-592.

18. Voir en particulier la systématisation, critiquée mais stimulante, proposée par le sociologue Y. BARel, La ville médiévale. Système urbain, système social, Grenoble, 1975.

19. Les formes de cette emprise sont un des thèmes majeurs abordés par N. Coulet, Aix-enProvence. Espace et relations d'une capitale (milieu XIV-milieu XV siècle), Aix-en-Provence, 1988. Ses méthodes ont ensuite été appliquées et affinées, par exemple dans divers travaux présentés dans une section ad hoc de La ville au Moyen Age. $120^{\circ}$ Congrès national des sociétés historiques et scientifiques, Section d'histoire médiévale et de philologie. Aix-en-Provence, 23-29 octobre 1995, dir. N. Coulet et O. Guyotjeannin, t. 1, Ville et espace, Paris, 1998.

20. Pour parler judicieusement d'espace, il ne suffit pas qu'un phénomène se déroule dans l'espace (!) - cc qui transformc cclui-ci en un simple " théâtre " - ni même qu'il y ait une organisation en étendue, mais il faut que cette extension en soit un élément essentiel.

21. C'est la conception vidalienne d'une géographie " absolue », science des lieux et non pas science des hommes, et l'on sait l'influence considérable qu'a eue Vidal de La Blache sur les historiens. 
par Fernand Braudel dans sa vaste synthèse sur l'économie mondiale, il est assez universel pour servir nos analyses spatiales, mais il est souvent employé pour des analyses de l'ordre du symbolique ou au contraire comme un descripteur du réel. Il convient notamment de ne pas le définir, dans son acception matérielle, comme un couple d'opposition (qui n'est qu'un simple instrument d'analyse arbitraire du réel) ${ }^{22}$, mais comme un phénomène de polarisation graduelle, depuis le centre vers la périphérie et inversement, impliquant des liens (certes dissymétriques) et une continuité entre les diverses parties ${ }^{23}$. On en a une image très concrète, pour les territoires locaux, dans l'organisation concentrique des finages à partir d'un noyau résidentiel aggloméré, avec une intensité de travail proportionnelle à la distance par rapport à la résidence.

Pourquoi, alors que la géographie sociale nous offre désormais des approches enrichissantes, les médiévistes l'ignorent-ils encore largement? À cause de barrières épistémologiques, mais plus encore, pensons-nous, pour des raisons pratiques, que nous aborderons ensuite. Bien sûr, on connaît l'argument classique : l'histoire prend en compte le temps, alors que la géographie étudie l'espace; mais on sait presque autant ce que cette conception a de fallacieux, puisque espace et temps sont aussi bien de simples catégories intellectuelles destinées à ordonner le chaos que le monde est pour l'homme. Il est certain que les études géographiques ont généralement une épaisseur chronologique faible, ce qui pose des problèmes de transposition $^{24}$; et les modèles sociologiques, indispensables pour appliquer ceux des géographes à la réflexion historique, souffrent des mêmes défauts. Concrètement, si les médiévistes sont peu tentés par la modélisation spatiale, même quand elle s'impose, les géographes étudiant l'espace

22. L'article de J. Le Goff, "Centre/périphérie ", Dictionnaire raisonné de l'Occident médiéval, dir. J. Le Goff et J.-Cl. Schmitr, Paris, 1999, p. 149-165, qui tente de généraliser ce concept à toutes les échelles et pour analyser de nombreux phénomènes, insiste plutôt sur l'antagonisme et la dissymétrie des deux éléments, dans un rapport domination/soumission ou action/réception, voire supérieur/inférieur. Ce rapport est réel en ce qui concerne les représentations de l'espace par les intellectuels (qui raisonnent beaucoup par dualisme), domaine de prédilection de l'auteur, mais il faut lui préférer la complémentarité en termes de fonctionnement spatial.

23. La définition qu'en donne Fernand Braudel est descriptive, fondée sur des oppositions entre niveaux d'efficacité économique à l'échelle mondiale : c'est une juxtaposition géométrique de zones concentriques, que l'on pourrait observer à n'importe quelle échelle et qui n'a rien de systémique. 24. On peut penser par exemple à l'œuvre de J. C. GARCia, O espaço medieval da Reconquista no Sudoeste da Perínsula Ibérica, Lisbonne, 1986, travail mćritoirc mais souffrant du susdit défaut méthodologique, regroupant une série de petites monographies historiques locales (urbaines) et supra-locales d'après le seul critère de la polarisation (théorie des lieux centraux), ce qui débouche sur une typologie régionale ignorant largement la problématique territoriale. 
social font rarement œuvre de médiévistes (sauf rare exception, comme les travaux de Roger Dion) ${ }^{25}$, et ils se contentent au mieux, méthodologiquement, de plaquer leurs schémas sur une matière historique abordée de deuxième main, tout en reconnaissant, épistémologiquement, que la répétition, donc l'épaisseur temporelle, est indispensable à la construction symbolique de l'espace.

\section{Problèmes pratiques dans l'articulation de la recherche en histoire/géographie}

Concrètement, que peuvent faire ensemble un médiéviste et un géographe, et quels sont les obstacles s'y opposant? Commençons, dans la tradition médiévale, par l'argument du précédent : il est possible d'associer médiéviste et géographe puisque certains l'ont fait!

\section{Des collaborations pionnières}

Dans un domaine (lusophone) qui est familier aux deux intervenants, on peut citer la conjonction épistémologique entre le géographe Jorge Gaspar et la médiéviste Maria José Lagos Trindade dans leur brève étude commune sur "L'utilisation agraire du sol dans la périphérie de Lisbonne au Moyen Âge et la théorie de von Thünen ${ }^{26}$ "; il est vrai que cette étude a eu pour cadre, en 1973, les " Journées de méthodologie appliquée des sciences historiques de Compostelle ", où toutes les audaces étaient permises et recherchées : les autres Européens, qui n'ont pas eu à hâter la fin du franquisme ou du salazarisme, n'ont pas eu le même besoin, politique, de ces audaces intellectuelles. On peut aussi citer la collaboration que mènent depuis plusieurs années les auteurs de ce propos autour d'un programme sur la territorialité.

Par ailleurs, même sans ces collaborations inter-personnelles, nombreux sont les historiens qui ont fait œuvre de géographie sociale depuis Lucien Febvre, avec La terre et l'évolution humaine, ou Braudel, avec sa

25. R. Dion, "La part de la géographie et celle de l'histoire dans l'explication de l'habitat rural du Bassin parisien ", Bulletin de la société de géographie de Lille, 1 (1946), p. 6-80, et son travail sur l'organisation de l'espace et l'endiguement de la vallée de la Loire au Moyen Âge, Histoire des levées de la Loire, Paris, 1961.

26. M. J. Lagos Trindade et J. Gaspar, "A utilização agrária do solo em torno de Lisboa na Idade Média ", Actas de las I jornadas de metodologia aplicada de la ciencias historicas, t. 2, Historia medieval, Santiago de Compostela, 1975, p. 89-94. 
Méditerranée, mais aussi depuis Les caractères originaux de Marc Bloch. $\mathrm{Au}$-delà des grandes synthèses historiques de certains géographes comme Roger Dion ou Jean-Robert Pitte sur le paysage agricole et les grands ensembles forestiers comme la Châtaigneraie, le travail spécifiquement médiévistique des géographes est moindre, mais on peut citer l'un d'eux, Vincent Clément, qui a fait œuvre de médiéviste pour sa thèse sur la forêt en Castille ${ }^{27}$.

Au-delà de l'argument du précédent, qui est plus une boutade qu'un argument recevable, les barrières ne manquent pas dans la recherche fondamentale - c'est-à-dire une fois dépassé le niveau du CAPES d'histoire-géographie $^{28}$. Passons - car cela nous mènerait à la caricature - sur les mésententes administratives et les représentations antagoniques mutuelles, qui ne sont guère différentes de celles, exposées plus longuement dans ce colloque par Luc Bourgeois et Isabelle Cartron, opposant historiens des " textes " et archéologues. Sauf heureuse exception (qu'expérimente une des signataires de cet article), les relations entre départements d'histoire et de géographie sont au mieux réduites, au pire exécrables, et la charge des " relations avec la géographie " est un pensum, souvent réservé au dernier recruté en histoire. En fait, le divorce épistémologique et académique, depuis trente ans, a engendré une séparation physique croissante : dans beaucoup d'universités créées ou déménagées depuis cette époque, les départements d'histoire voisinent et sont regroupés en UFR avec les sciences sociales, tandis que les départements de géographie sont installés dans des bâtiments de sciences dures ou d'économie et gestion. Cet éloignement se combine avec l'appartenance à des universités différentes (pour celles qui ont préservé les différences entre les anciennes facultés des lettres, sciences, droit, etc.) pour engendrer incompatibilités de calendriers, navettes d'étudiants, etc. Les divergences dans les préoccupations pédagogiques sont plus sérieuses : les historiens ne forment guère que des enseignants, alors que les études de géographie débouchent souvent sur l'expertise et la pratique concrète.

27. V. Clément, De la marche-frontière au pays des bois. Forêts, sociétés paysannes et territoires en Vieille-Castille (XY-XX' siècles), Madrid, 2002. Voir aussi J.-J. Dusors, "La place de l'histoire dans l'interprétation des paysages végétaux ", Mélanges de la Casa de Velázquez. Antiquité et Moyen Âge, 30 (1994), p. 231-251.

28. Pour les parties rédigées par St. Boissellier dans cet article, les connaissances des concepts et méthodes des géographes viennent en grande partie des ouvrages suivants : A. BAILly et R. FERras, Eléments d'épistémologie de la gégraphie, Paris, 1997; R. BRunet et O. Dollfus, Mondes nouveaux, Paris, 1990 (Géographie universelle, 1), une somme, par les tenants d'une géographie très "spatiale ", au sens géométrique; et Dictionnaire de la géographie et de l'espace des sociétés, dir. J. LÉvy et M. Lussault, Paris, 2003. 
Tout cela ne devrait guère arrêter les hommes de bonne volonté, comme en témoigne l'évolution du médiéviste coauteur de ces lignes, qui a été luimême un étudiant historien détestant la géographie...

\section{La question des sources et des techniques de traitement des données}

Il y a plus important. A priori, les méthodes n'ont rien à voir. Le géographe, ancré dans le présent et souvent voué à agir sur lui (en tant que praticien de l'aménagement du territoire), bénéficie des données et des méthodes de collecte de l'information engendrées par les sciences, les techniques et les pouvoirs politiques depuis le Xvir ${ }^{\mathrm{e}}$ siècle : le comptage y est essentiel, et il est efficient - contrairement à la quantification des données datant des ères pré-statistiques -, car la collecte y est exhaustive. Ces méthodes sont évidemment inaccessibles à l'étude des réalités matérielles médiévales, encore que certains archéomètres intégristes y prétendent. De ce point de vue méthodologique, la proximité du géographe sera plus grande avec le contemporanéiste qu'avec le médiéviste.

On peut noter toutefois que les sources administratives, notamment fiscales (comme les "estimes»), du bas Moyen Âge présentent une forme d'exhaustivité et offrent donc des possibilités statistiques, qui ont été plus exploitées par les historiens démographes que dans l'optique de l'analyse spatiale (sous prétexte que les transformations essentielles de l'organisation de l'espace seraient antérieures aux $\mathrm{XIII}^{\mathrm{e}}-\mathrm{XV}^{\mathrm{e}}$ siècles) : de véritables trésors documentaires attendent une analyse méthodique des localisations dont ils fourmillent. Par ailleurs, même sur la base de sources non chiffrées par elles-mêmes ni même chiffrables par l'historien, la simple mesure des distances est souvent le seul moyen de réfléchir quantitativement sur les structures matérielles médiévales; et il s'agit d'une quantification fiable, puisque aucun biais fonctionnel ou textuel n'est venu modifier, entre le Moyen Âge et nos jours, les distances que l'on mesure entre des objets matériels (à partir du moment où ceux-ci sont dûment localisés, sans le continuisme béat d'autrefois).

Sur le plan heuristique, il est certain que le géographe peut être rebuté par l'érudition nécessaire au médiéviste pour accéder à ses données, qu'elles soient écrites ou matérielles. La technicité de la démarche géographique n'est pas moindre (avec la statistique et la mise en carte), mais elle se concentre à une étape postérieure de son travail. La technicité disciplinaire n'est pas anodine, car elle constitue actuellement la principale barrière aux échanges entre disciplines : pour le géographe, comme pour ses collègues 
praticiens des autres sciences humaines et sociales, les médiévistes, plus que tout autre groupe d'historiens, apparaissent comme une sorte de secte au langage ésotérique. Pourtant, au lieu de croire que la pluralité et l'imbrication des juridictions sur les hommes à l'époque féodale constituent une monade, il suffirait que le médiéviste parle de cospatialité juridictionnelle au lieu de "mosaïque banale " pour que le géographe puisse expertiser la place du Moyen Âge central dans les diverses formes d'organisation de l'espace. En outre, le document de travail et parfois la synthèse consistent, pour le géographe, en un écrit iconographique, la carte, qui expose excellemment le fonctionnement systémique et les liens dialectiques, alors que l'exposé historique est de l'ordre du discours, qui est le vecteur privilégié de la relation causale.

Au-delà des problèmes heuristiques, on peut toutefois relever des éléments de méthodologie communs. Ainsi, l'usage de la méthode régressive dans l'analyse des réalités matérielles : bien sûr, pour le géographe, le recul dans le passé est un moyen de mieux comprendre le présent, alors que, pour l'historien, le départ depuis l'actuel ou un passé récent sert à atteindre le passé antérieur.

Recherches sur les liens espaceltemps : fascination et répulsion pour la modélisation et la généralisation des résultats

Ce problème du rapport avec le temps est autant technique qu'épistémologique. La tendance récente des géographes "physiciens " d'étudier l'action anthropique sur les paysages implique une prise en compte du temps " court " (c'est-à-dire non géologique), qui est un temps réellement historique (certes plus large que médiéval, comme le montrent les travaux interdisciplinaires pionniers du projet Archaeomedes, dans lesquels l'absence des historiens médiévistes « textologues " est navrante) ${ }^{29}$. Par ailleurs, après une époque où les médiévistes cherchaient à retrouver dans leur époque une typologie des paysages agraires élaborée sur le présent par les géographes ruralistes, les uns et les autres sont désormais bien conscients que la trame des villages et des terroirs d'Occident a connu au Moyen Âge des inflexions décisives et tellement durables qu'on ne peut les ignorer pour expliquer le "sub-contemporain".

29. Archaeomedes, Des oppida aux métropoles. Archéologues et géographes en vallée du Rhône, Paris, 1998. 
Plus fondamentalement, du point de vue strictement épistémologique, la géographie, toujours plus attirée par les sciences dures ou par celles qui prétendent l'être (comme l'économie), n'hésite plus à modéliser ses analyses et à proposer des concepts largement opératoires, sinon universels. En revanche, les médiévistes, écrasés par leur érudition, disent répugner à toute généralisation. En fait, l'ignorance persiste peut-être d'abord à cause de l'attachement des médiévistes à l'empirisme, chez des historiens qui se conçoivent volontiers comme des artisans dans leur atelier plutôt que comme des intellectuels. Or, les concepts géographiques ont une vocation à la généralisation et à la modélisation, qui heurte certains de nos collègues confondant la description du réel et les outils d'analyse ${ }^{30}$.

Plus généralement, les objets et les objectifs de l'analyse spatiale suscitent une méfiance dans les autres sciences humaines, parce qu'ils ont tendance à réifier les faits sociaux et parce qu'ils peuvent déboucher sur des modèles très spéculatifs, tels que les " chorèmes " de Roger Brunet ${ }^{31}$. C'est en fait entre sociologie et géographie que les notions de position, appartenance et distance peuvent être le plus facilement mises en commun. Les outrances néo-déterministes de l'analyse spatiale des phénomènes sociaux et culturels lui donnent auprès des autres sciences humaines l'image d'une science "dure». Si les historiens ont toujours récusé plus nettement l'idée même de "lois", partout le débat existe quant à la conception de ces «lois", soit comme des régularités signifiantes, soit comme des mécanismes absolus avec une capacité prédictive.

Mais on ne voit pas pourquoi les médiévistes récuseraient plus les « lois " géographiques que les modèles sociologiques ou économiques, dont ils font un large usage (parfois implicite et inconscient, il est vrai). Il est certain que l'espace, dimension mathématique, ainsi que l'environnement matériel, échappant partiellement au volontarisme social, se prêtent bien à l'énonciation de véritables normes axiomatiques, voire à la mise en équation,

30. Par un paradoxe qui n'est qu'apparent, c'est dans le domaine plus spéculatif de la "spatialisation" que la théorisation a été plus volontiers employée. Ainsi, le schéma centre-périphérie, très employé, comme on l'a dit, en histoire des représentations, est indispensable pour penser des processus matériels et sociaux complexes à l'échelle régionale, mais devient purement rhétorique dans certains emplois totalement abstraits - tels que la centralité hiérosolimitaine de l'Occident médiéval, qui n'existe que dans la spéculation des théologiens.

31. Un chorème est un néologisme géographique inventé par Roger Brunet en 1980. Le terme vient du mot grec chôra, qui signific territoire, étendue, lieu, contrée. C'est une représentation qui vise à montrer toute la complexité du territoire à l'aide de formes géométriques. Même s'il semble simplificateur, le chorème est réalisé de façon rigoureuse et il tiendra compte de toute la dynamique présente sur le territoire étudié. 
alors que la formulation des règles sociologiques reste plus proche du discours historien. Il n'en demeure pas moins que l'analyse de nos objets les plus traditionnels, comme la féodalité, est faite de plus en plus, comme en géographie, en termes de système et de combinaison de facteurs - les réseaux, les interactions, les interfaces -, tendant à la globalité et à la généralisation. De ce fait, par le biais du comparatisme, nous recherchons de plus en plus des structures dans ce qui est contingent, de la même façon que les géographes inscrivent l'étude régionale, exceptionnaliste, dans la géographie générale.

En fait, c'est évidemment le transfert et l'adaptation des concepts géographiques qui constituent le principal enrichissement. Prenons l'exemple des territoires locaux (paroisses, seigneuries juridictionnelles, terroirs), étudiés dans le cadre de monographies fondées sur la notion de communauté d'habitants. Cette échelle locale, qui est la seule pertinente en référence à des groupes humains (domaine socio-culturel), est à peu près l'équivalent en superficie du "géosystème " des géographes (quelques centaines à quelques milliers d'hectares). Quoique (et parce que) ce dernier, ne s'appliquant qu'aux paysages, soit défini par les géographes comme le cadre où se combinent les éléments du passé et les logiques d'auto-organisation de l'espace, on mesure, à cause de leur coïncidence territoriale, toute l'utilité qu'il y a à combiner la notion sociale de communauté locale et celle matérielle de géosystème.

Le principal problème est celui de l'adaptation. Pour prendre en exemple un domaine de recherche que nous avons un peu creusé au CESCM de Poitiers, celui de la territorialité, le concept spatial le plus employé, dans les travaux des médiévistes sur les territoires supra-locaux, est le réseau, mais il n'est pas sûr qu'il gagne à être généralisé. Dès lors que l’on parle en termes de lieux, la réticularité doit être maniée avec une grande précaution. Il en va ainsi des prétendus " réseaux " castraux, dans des régions d'atomisation féodale sans pouvoir planificateur, associés à une conception "stratégique " souvent anachronique, alors que c'est une forme de maillage de l'espace ${ }^{32}$. Ce concept, d'origine sociologique ${ }^{33}$, donc sans dimension

32. Nous employons ici le vocable "maillage " au " sens commun ", comme un ensemble de points (châteaux, églises, universités, etc.) - en fait un réseau -, ce qui est trompeur puisque les mailles sont, selon les géographes, les étendues polarisées à partir de ces points.

33. Concept élaboré dans les années 1930 par la psychologie sociale, puis généralisé en sociologie et anthropologie dans les années 1960 par les écoles de Manchester et d'Harvard, avant d'être introduit en géographie et enfin repris en histoire par les tenants de la micro-histoire (notamment le moderniste Giovanni Levi), afin de contourner les impasses de l'analyse structurale par l'analyse des relations. Voir par exemple A. Degenne et M. Forse, Les réseaux sociaux, Paris, 1994. Pour 
spatiale marquée (des échanges interpersonnels combinés entre eux, avec un fonctionnement d'ensemble), s'applique au lien social et se transpose assez mal aux structures matérielles ${ }^{34}$ : il ne peut y avoir réticularité des lieux que dans la mesure où ces lieux sont organisés fonctionnellement comme des relais, c'est-à-dire que les hommes y résidant échangent des informations et circulent de l'un à l'autre ${ }^{35}$. Les réseaux locaux médiévaux sont principalement les ports et marchés, les monastères d'un même ordre et, à la limite, les paroisses ${ }^{36}$ : les premiers constituent un réseau à cause de leur fonction de relais dans le commerce, qui est un système, et les autres à cause de leur intégration par une institution englobante ${ }^{37}$. Pour pouvoir parler d'un réseau de lieux, il faut en outre définir précisément la nature des liens et ne pas se contenter de parler vaguement de relations. Or, ces liens sont plus complexes que les chaînes d'interconnaissance fondatrices des réseaux personnels : sujétion commune à une autorité - dans ce cas-là, il y a une centralité -, complémentarité fonctionnelle, contiguïté spatiale (impliquant les relations d'" interface " des géographes) ou contacts réels (une véritable interconnexion avec circulation d'hommes, comme dans le cas de l'envoi de podestats dans l'Italie communale ou des relations entre communautés juives).

voir ce que peuvent néanmoins apporter les géographes à l'analyse des réseaux, on peut se reporter aux actes du Festival de géographie de Saint-Dié 2005 consacré à ce thème "Le monde en réseaux : lieux visibles, liens invisibles ", cf. http://fig-st-die.education.fr/actes/actes_2005/index.htm.

34. La représentation graphique des réseaux sociaux, comme d'ailleurs de toute autre position et interaction sociale, est particulièrement trompeuse, car elle donne l'illusion d'une spatialité (notamment par les maillages qu'elle crée), alors qu'elle représente des " positions " en fait immatérielles - et c'est le problème de la notion même d' "espace social " dans son emploi par certains sociologues; on peut voir toutes les ambiguïtés de cette méthode dans M. GRIBAUDI, "Les discontinuités du social. Un modèle configurationnel ", Les formes de l'expérience, dir. B. LepeTIT, Paris, 1997, p. 187-225.

35. En histoire, ces liens interpersonnels ont été étudiés surtout du point de vue de l'administration, des communautés religieuses, des familles et des milieux intellectuels, principalement par la méthode prosopographique.

36. Nous ne parlons pas ici des réseaux totalement matérialisés, qui existent soit à une échelle infralocale (les structures d'irrigation et les parcellaires bâtis et non bâtis), soit à une échelle beaucoup plus vaste (les routes).

37. Récemment, un article d'Annie Saunier examine, à propos des hôpitaux en France, les notions de réseau et de système hiérarchique, cf. A. SAUNIER, "La trame hospitalière médiévale : hiérarchie ou réseau? ", Hôpitaux et maladreries au Moyen Âge : espace et environnement. Actes du colloque international d'Amiens-Beauvais 22, 23 et 24 septembre 2002, Amiens, 2004; la majorité des articlcs dc cc colloque examinent plutôt l'effet de centralité de ces institutions dans le tissu urbain, mais certains étudient les relations spatiales entre ces lieux à l'échelle régionale et, plus pertinemment, dans le cadre d'un ordre religieux. 
On aura bien compris, aux propos qui précèdent, que notre revendication est - sans aucune originalité - une histoire médiévale refusant le "sens commun " et dégagée des belles-lettres (malgré la dimension incontestablement littéraire de nos sources), et ceci par l'utilisation des concepts et, éventuellement, des méthodologies des autres sciences. Il est invraisemblable que l'histoire de l'art et celles du droit, des sciences et de la musique constituent des disciplines académiquement distinctes de l'histoire au nom de leur technicité, et que l'historien des faits sociaux ou économiques ne se considère pas comme un praticien (si ce n'est un théoricien) de la sociologie, de l'anthropologie, de l'économie et bien sûr de la géographie, sans aucunement perdre son identité pour autant. Une méthode géographique chez les médiévistes et une prise en compte du temps long en géographie sociale : alors, les histoires d'amour finissent mal? Seulement en général...

\author{
Nacima BARON
}

Université Paris-Est Marne-la-Vallée

LVMT (INRETS, UMR-T 9403)

Stéphane BoISSELlier

Université de Poitiers

CESCM (CNRS, UMR 6223) 